\title{
Wake-blade interaction in steam turbine stages
}

\author{
Jerzy Świrydczuk, Prof. \\ Institute of Fluid-Flow Machinery, Gdansk, Poland
}

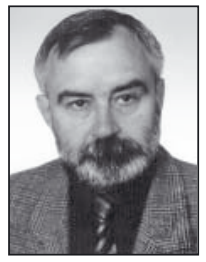

\begin{abstract}
The article discusses the phenomenon of stator Wake/Rotor cascade (W/R) interaction in a steam turbine stage, and the ability to capture it in turbine stage design calculations making use of standard numerical codes. Firstly, the $W / R$ interaction is analysed by comparing its real, experimentally recorded course with the numerical results obtained using vortex theory models and methods. This part of the analysis ends with formulating a conclusion about stochastic nature of the W/R interaction and indicating its reason, which is the vortex structure of the stator wake. Next, a question is discussed whether and how this stochastic nature of the examined phenomenon can be taken into account in calculations of Reynolds Averaged Navier-Stokes (RANS) equations. Differences are indicated between the uniform pattern of the stator wake obtained using a RANS code and the vortex structure of the real wake. It is concluded, however, that despite these differences the RANS results correctly reflect the time-averaged course of the real $W / R$ interaction, and the process of averaging the flow parameters on the sliding plane between stator and rotor calculation areas can be treated as sort of "numerical averaging" of different realisations of the W/R interaction.
\end{abstract}

Key words: turbine passage; secondary vortices; stochastic behaviour; CFD calculations

\section{SECONDARY VORTEX STRUCTURES IN A TURBINE ROW}

Steam turbines are widely used in shipbuilding industry as the main engines on ships which need huge driving power (large super-tankers, for instance), or the nature of their operation is characteristic for long-distance sailing without calling at a harbour (ice-breakers or marine submarines with nuclear propulsion systems). Permanent competition between turbine producers forces them to offer high-quality and highefficiency products, the working characteristics of which are to be presented to the buyer at an earliest possible stage of negotiations. These characteristics are to be calculated very precisely, as delivering the product which does not meet parameters named in the contract would result in fines paid by the producer. On the other hand, when the sold turbine reveals the real power output or efficiency much higher that that declared in the offer, it means an extra bonus for the buyer, or, looking from the other side, loss for the seller who could have sold it for a higher price. That is why steam turbine designers attempt to calculate basic turbine parameters very accurately, taking into account as many factors as possible. One of these factors which were given special attention in recent years is the effect of interaction of the secondary vortices forming in turbine blade stator and rotor passages on the level of the generated stage loss.
The flow through a steam turbine stage is extremely complex, Fig. 1 [1]. Even in high pressure stages of relatively simple radial geometry in which the flowing superheated steam has physical properties close to those of the perfect gas, a number of secondary vortices, both forming in stator or rotor cascades and approaching from upstream rows, can be observed. Looking from the cascade front, two pairs of horseshoe vortices 1 and 2 are formed at the leading edge of each blade, one pair near the hub endwall and one near the tip endwall. Then, inside the rotor passage these vortices mix, partially or entirely, with the passage

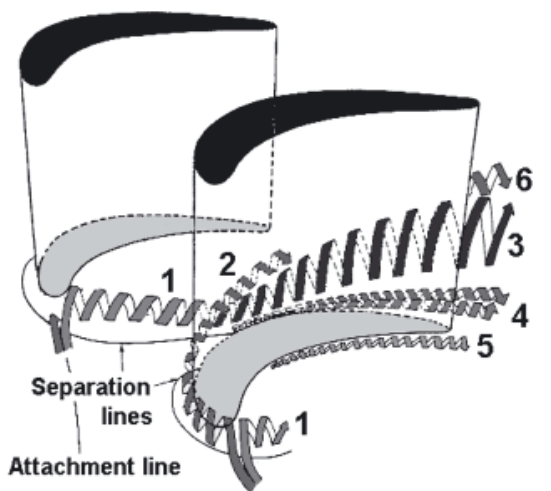

Fig. 1. Secondary flow structure in turbine stage: 1, 2) pressure and suction side horseshoe vortices, 3) passage vortex, 4, 5) suction and pressure side corner vortices, 6) trailing shed vortex 
vortices 3 generated by the passage cross flows. In the corners between the blade hubs, or tips, and the passage endwalls, the action of the passage vortices leads to the formation of corner vortices 4 and 5 . The rear parts of the stator and rotor blades are the sources of wakes which shed from their trailing edges and move downstream into next turbine parts. The interaction of the passage vortex with the blade trailing edge is believed to be a source of a so-called trailing shed vortex 6 , having the rotation opposite to that represented by the passage vortex [2]. Flow separations, occasionally observed at rotor blade surfaces, can lead to the creation of large-scale vortices of various orientations. And last but not least, leakage flows over the blade tips in the rotor cascades usually generate characteristic vortex structures the intensity of which depends on the adopted type of sealing systems.

The scale and direction in which the interaction of all above vortices affects turbine performance parameters is not clear. Generally it is assumed that neglecting this phenomenon leads to underestimation of stage losses [3], but opinions can also be found that in some cases the presence of vortex structures improves the stage performance by periodical sweeping out the stagnation areas.

Although extremely complex, the abovementioned vortex interaction in a steam turbine stage can be successfully analysed using Computational Fluid Dynamics (CFD) codes which solve the Reynolds Averaged Navier-Stokes (RANS) equations in computational domains of arbitrary geometry. It should be stressed, however, that these CFD calculations require extremely fine grids to take into account the widest possible range of small-scale vortices. A commonly accepted approach here is to perform the calculations in the geometry consisting of one stator and one rotor passage in combination with special procedures taking into account the relative rotor/stator motion when passing the flow field parameters between these two computational domains. The assumption which is believed to justify this approach is that all unsteady phenomena take the identical course in each stator and each rotor passage.

However, there is one exception to this assumption, which is the interaction of the wakes shedding from the stator blade trailing edges with the downstream rotor blade cascades, the W/R interaction. A detailed analysis of this phenomenon and its effect on the turbine stage design process is the basic subject of this article, which synthesises the experience gained by the author in this area over the recent decade. Due to the lack of reliable experimental data on $\mathrm{W} / \mathrm{R}$ interaction coming from marine steam turbines, in order to keep the geometrical and physical conditions of the analysis within realistic regimes the geometry of the cascade used in the analysis was assumed identical with the midspan cross-section of a real HP impulse turbine rotor in

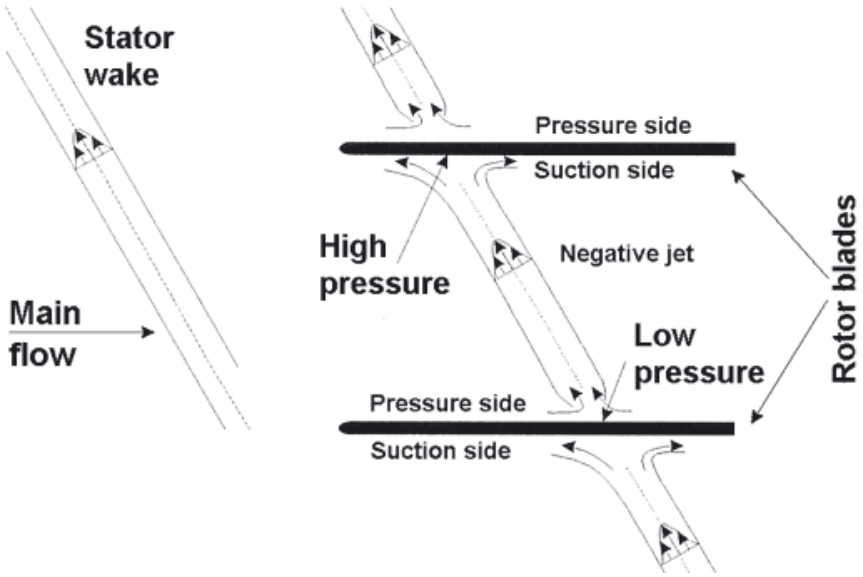

Fig. 2. Negative jet concept of wake model operation in one of inland power plants in Poland. The selected parameters of the flow through the rotor cascade were also taken from the measurements performed in this turbine. The conclusions resulting from the presented analysis are valid for both inland and marine applications of steam turbines.

\section{WAKE MODELLING}

In the past, the wakes shedding from turbine blades were modelled in various ways depending on a current state of knowledge on the phenomenon. In one of the first models proposed by Meyer in 1958 [4] the wake was represented by a regular band of velocity deficit, a so-called "negative jet", immersed in the otherwise uniform flow, Fig. 2. The stator wakes were transported with the main flow and chopped into linear segments by the rotor blades. Within the rotor passage the wake segments, keeping their linear shapes, generated secondary velocity fields responsible for fluid transport from the pressure side to the suction side of the passage.

Due to a very simplified geometry assumed for both the stator wake and the rotor blades, the Meyer's model could not take into account any possible deformation of the stator wake on its way through the rotor. However, as was noticed by Smith [5], the velocity difference between the rotor blade pressure and suction sides in a real turbine row makes the opposite ends of the wake segment move with different velocities, which leads to stretching and shearing of the entire segment. Consequently, when the wake leaves the rotor, its structure resembles a saw rather than the initial linear and continuous shape.

Taking into account the wake deformation was only possible after the development of computer technology and the appearance of CFD codes which solve the fluid flow equations in two and three dimensions. Initial attempts to capture the wake deformation based on the potential flow model with a continuous vortex layer of changing intensity shed from the blade trailing edge, which was an adaptation of the classical Prandtl's concept of unsteady flow over an airfoil [6] to the cascade geometry $[7,8]$. Once shed to the flow, successive wake segments moved with local flow velocities through the rotor cascade, thus making a further contribution to unsteady flow effects in this area, Fig. 3. Instantaneous intensities of the vortices, being the response to changing flow conditions in the vicinity of blade trailing edges, were calculated from a set of integral equations.

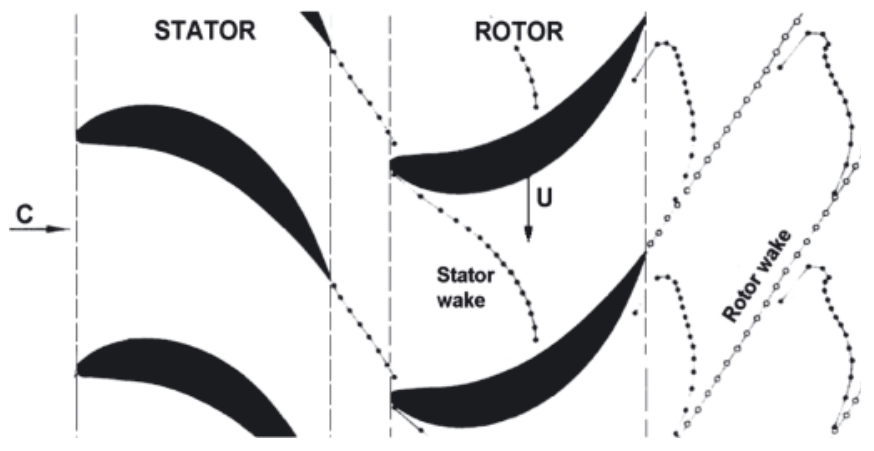

Fig. 3. Vortex wake model

It is noteworthy that despite a discrete representation, the wakes in these models were still interpreted classically as continuous bands of fluid in otherwise inviscid and incompressible flow, with the velocity deficit changing uniformly along the wake axis.

More advanced studies of the stator wake behaviour in the rotor cascade were possible using specialised 3D codes developed to solve RANS equations for turbulent, compressible, and viscous flows through fluid-flow machines. 
In the calculations performed with the aid of those codes the stator wakes were obtained as part of the solution and their characteristics did not have to be assumed a priori. The RANS codes enabled studying numerous aspects of W/R interaction, including wake deformation [9], profile losses [10], unsteady heat transfer [11], boundary layer transition [12], pressure fields and blade load fluctuations [13], etc. Experimental verification of the obtained results was, generally, good - at the level determined by the accuracy and resolution of the measuring devices used in those times.

The initial calculations performed with the aid of 3D RANS codes made use of grids of relatively moderate resolution. As a result, the generated wakes had the forms of regular layers of increased entropy and decreased flow velocity, without visible traces of inner structure, Fig. 4. However, the experiments oriented on taking more detailed insight into the stator wakes revealed clear frequencies in the signals recorded in the wake area, which testified to the presence of regular fine vortex structures in there $[14,15]$. More recent numerical computations performed for isolated stators using sufficiently fine grids and time steps also produced wake patterns in which two rows of well developed vortices having the structure similar to that of the von Karman vortex street was observed $[16 \div 19]$. In this context, a question should be raised about the consequences brought by the presence of active vortices in the wake to conventional CFD analyses making use, as a rule, of RANS codes as basic tools.

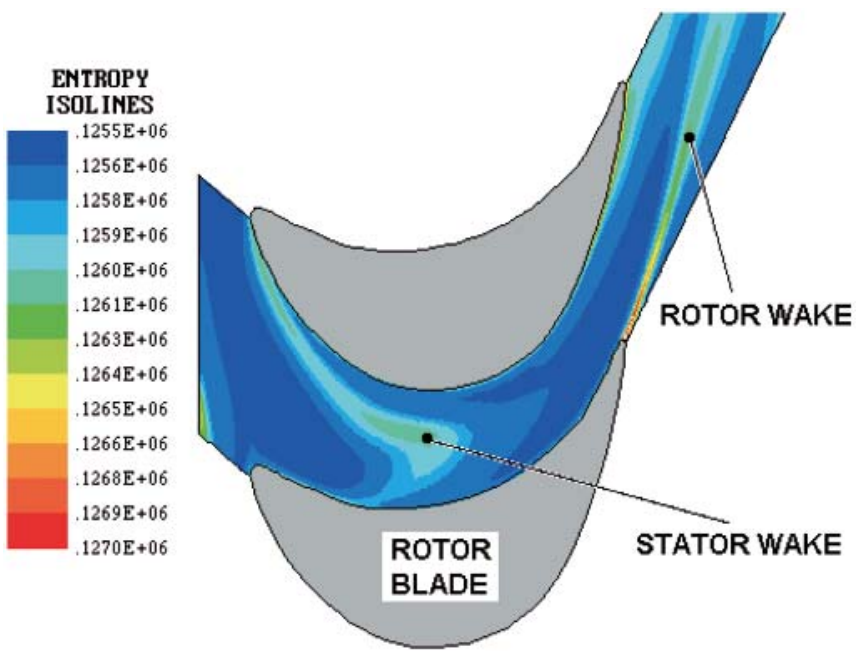

Fig. 4. Stator wake deformed inside the rotor passage - NS calculations

\section{VORTEX STRUCTURE OF THE WAKE AND ITS CONSEQUENCES}

Analysing numerically the development and interaction of the vortex stator wake with the rotor cascade is much more difficult that in case of all other secondary structures described in section 1. Particular wake segments chopped off by the rotor blades do not have, in general, the same characteristics, i.e. do not consist of vortices of identical strengths and positions at the time of chopping. And any differences in the initial structure of the chopped-off wake segment result in inevitable differences in its further motion through the rotor passage. Unlike the passive band-like wakes observed in conventional CFD calculations, the sample of which is shown in Fig. 4, active vortices interact both with each other and with the boundaries, which makes their trajectories differ from those estimated on the basis of pure main flow convection.

The initial arrangement of the vortices in the wake segment at the time of chopping-off by the rotor blade may be described using a so-called phase shift, $\varphi$, which defines the range of displacement of the von Karman vortex structure along the wake axis. The definition of the phase shift is shown in Fig. 5 where it is interpreted as the ratio of the distance $s$ between corresponding vortices in two different von Karman wake vortex segments $a$ and $b$ to the distance $l$ between two adjacent vortices of the same rotation in one segment.

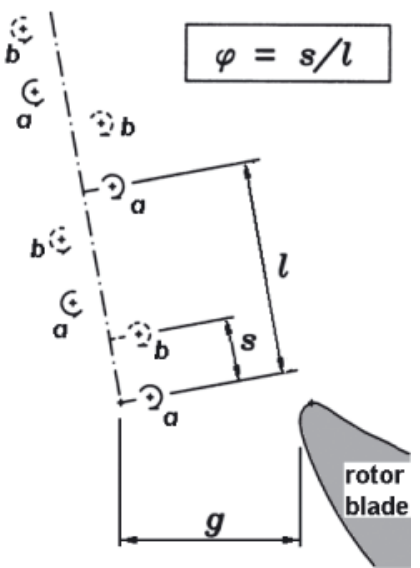

Fig. 5. Phase shift in the stator wake vortex arrangement

The above aspect of the W/R interaction cannot be effectively analysed using a NS code. Due to possible differences in phase shifts of the particular chopped-off wake segments we cannot assume any longer that their behaviour inside each rotor passage is the same. Consequently, to capture the complete W/R interaction and its effects we should generate a computational grid covering all stator and rotor blade passages. And what is even more challenging, this grid should be fine enough to detect and study the behaviour of such small structures as the wake vortices. Keeping in mind that the number of passages in the stator or rotor row can exceed a hundred in a real turbine, the creation of a sufficiently fine grid in those circumstances is beyond capabilities of even most powerful computing centres.

That is why studying vortex related aspects of the W/R interaction needs a flow model which would take into account the differences between vortex arrangements in individual wake segments. The model applied in the present study makes use of the Vortex Dynamics Theory (VDT) which defines principles of motion of vortices in multiply connected regions [20]. Along with the ability to model arbitrary phase shifts of the wake vortex configurations, VDT provides several invariants of vortex motion which can be used as additional tools to increase the accuracy of flow calculations. The here applied VDT model bases on the 2D potential flow through an infinite cascade of blades, with the wake represented by vortex singularities. Unlike the earlier works making use of the vortex model $[7,8]$, in the present study each singularity represents an individual coherent vortex.

\section{FLOW MODEL}

A procedure which is frequently applied in studying unsteady vortex phenomena bases on generating, step by step, a series of instantaneous vortex configurations to illustrate the course of the phenomenon. In this procedure each previous configuration is used as the input data for calculating the next one. Therefore of crucial significance for the accuracy of the final result is the accuracy with which transient configurations are calculated.

In most typical vortex model applications in blade flow analyses the shape of the blade is modelled using a sequence 
of discrete vortices representing the bound vorticity distributed along the blade contour - see, for instance, Basu and Hancock for a single profile [21], or Lienhart [7], and Krammer [8] for a cascade. The above blade description can be a source of relatively large inaccuracy in evaluating instantaneous velocities of free vortices, especially those moving close to the blade contour. To reduce this inaccuracy, the present study makes use of conformal mapping [22] and the circle theorem based inversion technique [23] which move all singularities used for defining the blade shape off the boundaries, thus creating a continuous and regular velocity field in the entire flow area. A detailed procedure of constructing the stream function for a system of blades in various configurations can be found in Refs. [24 $\div 28]$. The accuracy of the solutions obtained using the conformal mapping is believed to converge asymptotically to the accuracy of the analytical solutions.

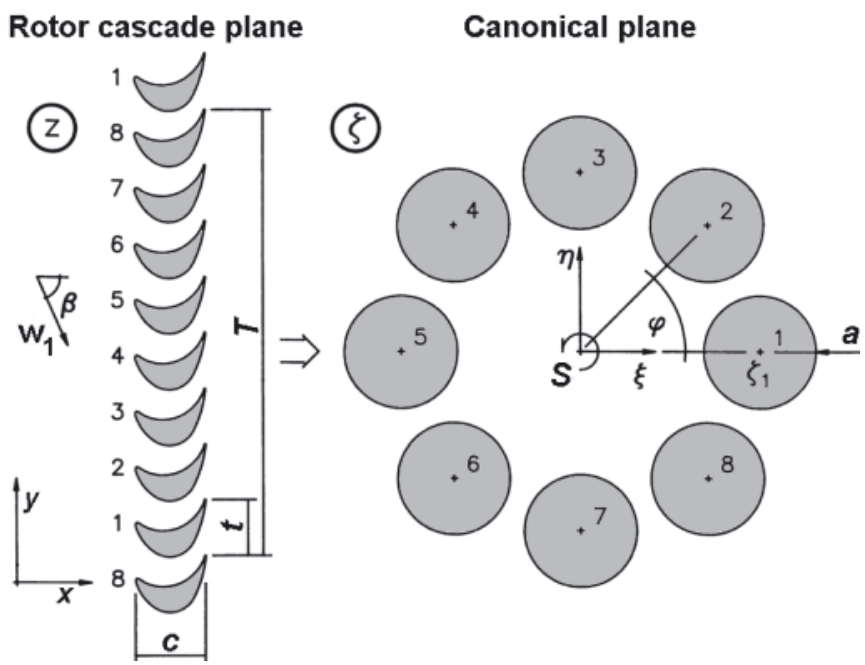

Fig. 6. Infinite blade cascade mapped into a circular cascade of circles

The mapping function used in the present analysis maps a K-element segment of an infinite linear cascade of blades into a circular cascade of $\mathrm{K}$ circles, Fig. 6. A general formula for this function has the form of an infinite fraction series [22]:

$\mathrm{z}=\frac{\mathrm{T}}{2 \pi} \ln \left[\zeta+\sum_{\mathrm{k}=1 \mathrm{n}=1}^{\mathrm{K}} \sum_{\mathrm{n}}^{\infty} \mathrm{e}^{\mathrm{i}(\mathrm{n}+1)(\mathrm{k}-1) \phi}\left(\frac{\mathrm{a}}{\left.\zeta-\zeta_{\mathrm{l}} \mathrm{e}^{\mathrm{i}(\mathrm{k}-1) \phi}\right)^{\mathrm{n}}}\right]\right.$

The number $\mathrm{K}$ of the blades composing the transformed cascade segment can be chosen arbitrarily. The resultant length of the cascade segment is $\mathrm{T}=\mathrm{tK}$, where $\mathrm{t}$ is the cascade pitch. In numerical applications the infinite sum of mapping coefficients is reduced to a limited number, $\mathrm{N}$, the choice of which compromises between the required accuracy and the available computation time. Unknown parameters in Eq. (1) are the centre of the first circle $\zeta_{1}$, the circle radius a, equal for all circles, and a series of complex coefficients $\mathrm{C}_{\mathrm{n}}$. For the analysis performed in the rotor reference frame these parameters are to be calculated once in the entire flow calculation process.

In the canonical plane, $\zeta$, the parallel flow transformed from the rotor cascade plane, $\mathrm{z}$, is represented by the source $\mathrm{Q}_{\mathrm{r}}$ and the vortex $\Gamma_{\mathrm{r}}$ located at the origin $\mathrm{S}$. The strengths of these two singularities are related with the assumed inlet flow velocity, $\mathrm{w}_{1}$, and inlet flow angle, $\beta$, in the physical plane and are calculated as:

$$
\Gamma_{\mathrm{r}}=\frac{\mathrm{w}_{1} \mathrm{~T}}{2 \pi} \cos \beta
$$

for the vortex and:

$$
\mathrm{Q}_{\mathrm{r}}=\frac{\mathrm{w}_{1} \mathrm{~T}}{2 \pi} \sin \beta
$$

for the source.

A general formula for the complex flow velocity in the canonical plane $\zeta$ is also given in the form of a fraction series [22]:

$$
\begin{gathered}
\overline{\mathrm{v}}(\zeta)=\frac{\mathrm{Q}_{\mathrm{r}}-\mathrm{i} \Gamma_{\mathrm{r}}}{\zeta}-\sum_{\mathrm{l}=1}^{\mathrm{L}} \sum_{\mathrm{s}=1}^{\mathrm{S}}\left[\frac{\mathrm{i} \Gamma_{0}}{\zeta-\zeta_{\mathrm{s}, \mathrm{l}}^{+}}-\frac{\mathrm{i} \Gamma_{0}}{\zeta-\zeta_{\mathrm{s}, \mathrm{l}}^{-}}\right]+ \\
\quad-\sum_{\mathrm{k}=1}^{\mathrm{K}} \sum_{\mathrm{w}=1}^{\mathrm{W}} \frac{\mathrm{i} \Gamma_{\mathrm{w}, \mathrm{k}}}{\zeta-\zeta_{\mathrm{w}, \mathrm{k}}}+\sum_{\mathrm{k}=1}^{\mathrm{K}} \sum_{\mathrm{n}=1}^{\mathrm{N}} \mathrm{D}_{\mathrm{k}, \mathrm{n}}\left(\frac{\mathrm{a}}{\zeta-\zeta_{\mathrm{k}}}\right)^{\mathrm{n}}
\end{gathered}
$$

Here $D_{k, n}$ are unknown complex coefficients calculated from the system of equations expressing the condition of zero normal velocity along the entire contour of each blade, and zero tangent velocity at circle points corresponding to the blade trailing edges. These coefficients represent the reaction of the circles to both steady and unsteady flow components. $\mathrm{S}$ is the number of pairs of vortices in a single stator wake, and $\Gamma_{0}$ is the strength of the positive vortex in the stator wake, a significant parameter in the present study. $\zeta_{\mathrm{s}, 1}^{+}$and $\zeta_{\mathrm{s}, 1}^{-}$are instantaneous locations of the positive and negative vortex, respectively, in the 1-th stator wake. $\mathrm{W}$ is the number of vortices in one rotor wake and $\zeta_{\mathrm{w}, \mathrm{k}}$ is an instantaneous location of the rotor wake vortex generated in the w-th step downstream of the k-th blade. All those parameters are to be calculated at each step of computation.

The flow velocities calculated in the canonical plane are transformed to the physical plane using the formula [22]:

$$
\mathrm{v}(\mathrm{z})=\frac{\mathrm{v}(\zeta)}{\frac{\mathrm{dz}}{\mathrm{d} \zeta}}
$$

where $\mathrm{dz} / \mathrm{d} \zeta$ is calculated from Eq. (1).

The velocity $v_{i}$ of the $i$-th vortex situated at point $\zeta_{i}$ in the canonical plane (here $i$ stands for all indices of both stator and rotor vortices) can be calculated from Eq.(4) using the Helmholtz and Kirchhoff's principle that the vortex does not act upon itself [23]:

$$
\overline{\mathrm{v}}_{\mathrm{i}}\left(\zeta_{\mathrm{i}}\right)=\overline{\mathrm{v}}\left(\zeta_{\mathrm{i}}\right)-\lim _{\zeta \rightarrow \zeta_{\mathrm{i}}} \frac{\mathrm{i} \Gamma_{\mathrm{i}}}{\zeta-\zeta_{\mathrm{i}}}
$$

The vortex velocities are transformed from the canonical plane to the physical plane using the formula [23] :

$$
\overline{\mathrm{v}}_{\mathrm{i}}\left(\mathrm{z}_{\mathrm{i}}\right)=\overline{\mathrm{v}}_{\mathrm{i}}\left(\zeta_{\mathrm{i}}\right) /\left.\frac{\mathrm{dz}}{\mathrm{d} \zeta}\right|_{\zeta_{\mathrm{i}}}-\left.\frac{\mathrm{i} \Gamma_{\mathrm{i}}}{2} \frac{\mathrm{d}^{2} \mathrm{z} / \mathrm{d} \zeta^{2}}{[\mathrm{dz} / \mathrm{d} \zeta]^{2}}\right|_{\zeta_{\mathrm{i}}}
$$

The derivatives in Eq. (7) are calculated from Eq. (1).

In the VD Theory, the strengths and initial positions of the wake vortices are to be assumed a priori based on the available information on the examined flow, including in particular the scale of the velocity deficit generated by the wake and the width of the wake. Unfortunately, the literature on the subject gives little and rather incomplete data on the inner structure and strength of the vortex wakes in any turbine stages. In this situation, in order to obtain the wake characteristics which could be considered reliable and representative for the examined turbine flow, in the reported study the initial wake 
parameters were reconstructed from preliminary 3D RANS calculations done using a specialised code FlowER for the stage being the prototype for the rotor geometry examined in the present study.

\section{STEADY FLOW TESTS}

Before the basic part of the analysis, the applicability of the VDT flow model to the examined phenomenon of vortex interaction inside the turbine rotor passage was checked on a series of comparison tests performed using the results of the FlowER calculation as the reference.

The first test consisted in comparing the velocity distributions inside the rotor passage obtained using the potential flow model, without vortices, and FlowER. The input data for the potential flow calculations were the rotor inlet velocity $\mathrm{w}_{1}$, and angle $\beta$, computed by FlowER and averaged at the rotor inlet midspan. The results of the test are shown in Fig. 7 as velocity modulus and angle distributions in selected passage sections located at: $\mathrm{x} / \mathrm{c}=-14.85 \%, 25.73 \%, 66.52 \%$ and $101.68 \%$ from the rotor blade leading edge. Bars in the diagrams represent the FlowER data, while the points located close to their tops stand for the results obtained using the potential flow model. The presented data well match each other, except for the rotor blade boundary layer near the blade suction side and the rotor wake where the viscous effect are dominating.

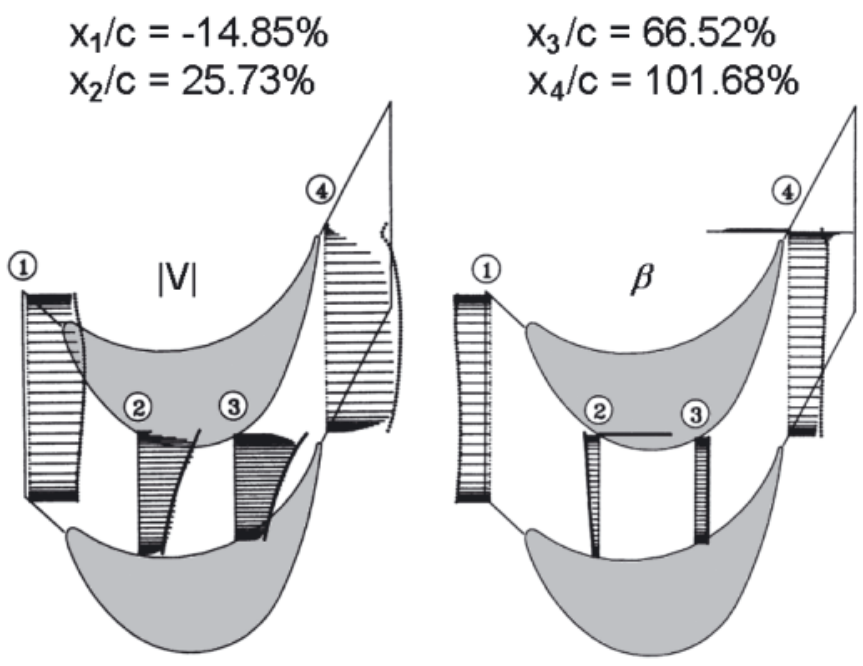

Fig. 7. Distributions of steady flow velocity modulus (left) and angle (right) in rotor passage

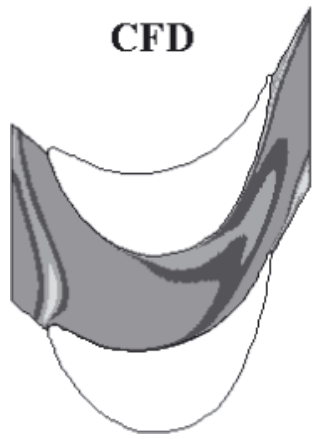

POTENTIAL

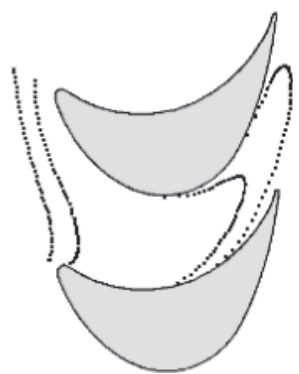

Fig. 8. Stator wake deformation in the rotor passage: 3D CFD midspan solution (left) and potential flow model (right)

The second test was oriented on comparing instantaneous images of wakes computed by FlowER with those obtained using the potential model. A sample FlowER wake is shown in Fig. 8 (left). The corresponding potential model of the passive wake, $\Gamma_{0}=0$, was generated by placing two parallel lines of fluid particles upstream of the rotor and then allowing them to move with the flow through the rotor. Two instantaneous locations of these pairs of lines, selected so as to correspond to the wake locations generated by FlowER, are shown in Fig. 8 (right). The agreement between the both curves is surprisingly good, bearing in mind complex structure of the flow in a turbine stage. These two tests testify to good credibility of the potential model to determine accurately the velocity fields inside the rotor passage, and thus to create a realistic pattern of the wake motion through it.

\section{THE W/R INTERACTION}

Following the standard procedure mentioned in the section entitled Flow model the course of the stator wake/rotor cascade interaction was studied by calculating a sequence of instantaneous flow patterns showing current distributions of stator wake vortices inside the rotor passage. The input parameters for these calculations were: the inlet flow velocity $\mathrm{w}_{1}$, the inlet flow angle $\beta$, and the strengths of the stator vortices $\Gamma_{0}$. The main variable parameter of the study was the phase shift factor, $\varphi$, defined in Fig. 5 (the basic distribution of the wake vortices, corresponding to $\varphi=0.0$, is labelled "a" in this figure).

Figure 9 shows a selection of these flow patterns covering one period of wake passing. The flow is from left to right. Stator wake vortices are represented in the diagrams by small circles, green and red for the positive - counter-clockwise and negative - clockwise rotation, respectively. Black circles downstream of the rotor blades mark initial segments of the rotor wake. The diagrams also include two sequences of small black dots representing hypothetical positions of the stator wake vortices in case they moved passively with the potential flow velocity, like the fluid particles in Fig. 8, right.

In the initial diagram, step $\mathrm{N}$, the wake has just approached the cascade and is being cut apart by the blade. So far the only noticeable effect of activity of the vortices is the reduced velocity of their motion, compared to the potential flow velocity (compare the positions of the active vortices and the corresponding passive dots). The reduced velocity of motion of the von Karman vortex street in the flow, resulting from an additional velocity component induced by the street on itself, was first proved, both theoretically and experimentally, by von Karman [32], and its presence here testifies to the correctness of wake motion modelling. The next diagrams, steps $\mathrm{N}+0.2 \mathrm{~T}$ and $\mathrm{N}+0.4 \mathrm{~T}$, show early stages of wake deformation. Negative vortices composing the central part of the wake segment are accelerated, while those situated close to its end are decelerated. An opposite tendency is observed in the motion of the positive vortices. As a result, the outer vortices of the two rows come closer to each other while the central ones become more distant. All this leads to noticeable segregation of vortices representing different rotation signs, with their further motion in two nearly isolated groups, visible in the final diagrams, steps $\mathrm{N}+0.6 \mathrm{~T}$ and $\mathrm{N}+0.8 \mathrm{~T}$. Of these two groups, only the second group of vortices moves approximately with the velocity predicted by FlowER for the entire wake, while the first group moves faster, contributing to the growing rate of deformation of the inner structure of the wake.

Beside the basic variant, $\varphi=0$, the study included three more variants of wake vortex distribution, which were denoted by phase shifts, $\varphi$, equal to $0.25,0.5$, and 0.75 . The above described segregation of a one-sign vortices and their further motion in nearly isolated groups were recorded in all cases. The most spectacular differences were observed in the motion of the vortices located close to the cut-off area. These vortices took visibly different trajectories, which in extreme cases led to the separation of isolated vortices from the wake. 


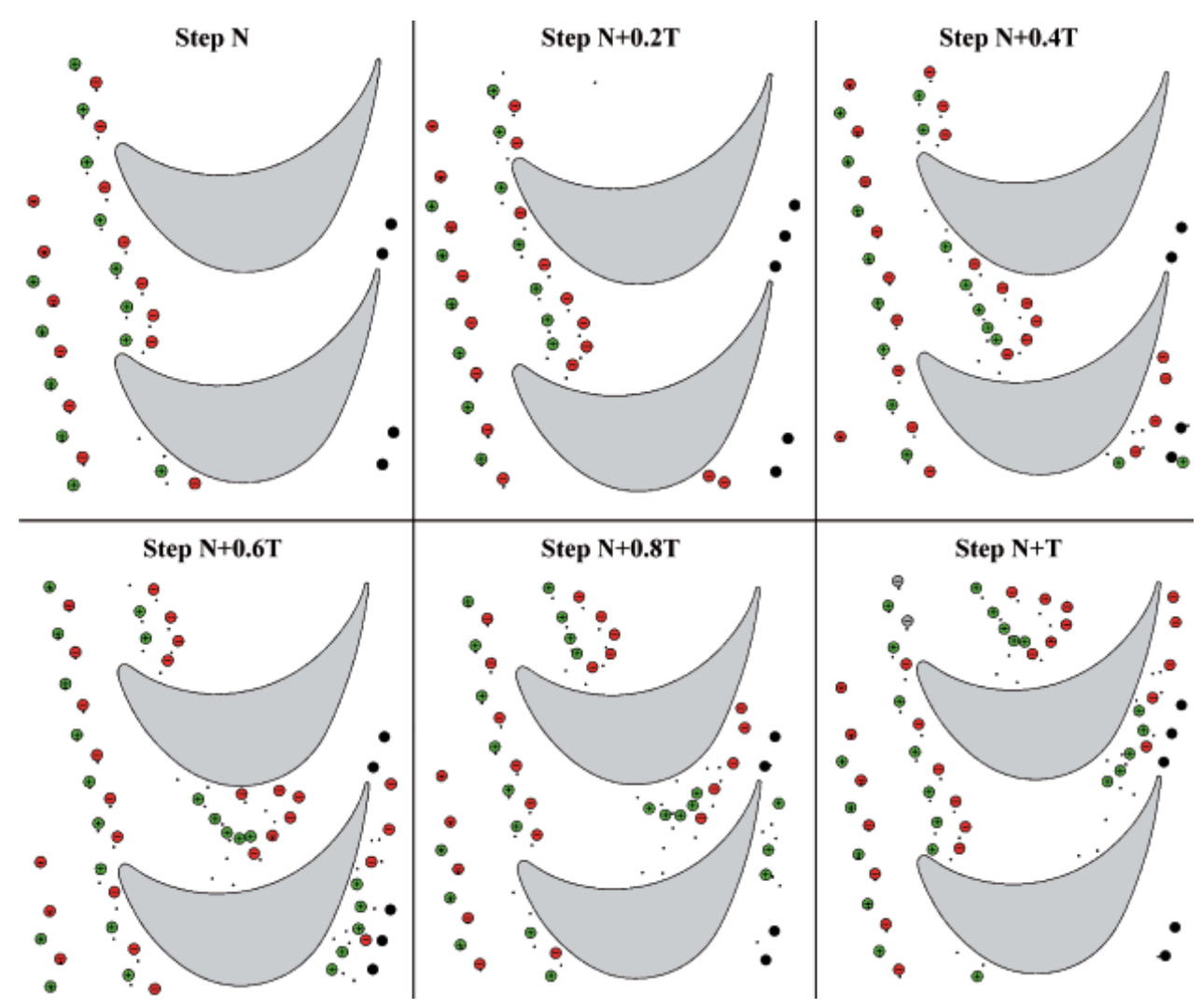

Fig. 9. Stator wake deformation in the rotor passage: $\Gamma^{*}=0.005 ; \varphi=0.0$

Characteristic wake configurations with isolated vortices are shown in Fig. 10.

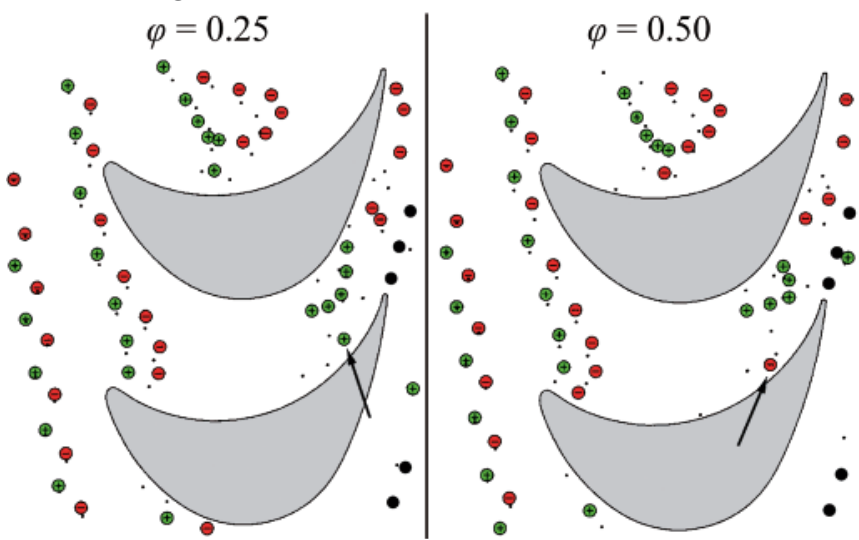

Fig. 10. Extreme cases of wake deformation. Step $N+T$ : separation of isolated positive vortex (left) and negative vortex (right)

The above result prove and clearly illustrate the existence of mechanisms in the flow which make the same stator wake behave in a different way in the rotor passage only because of a different phase shift in the initial vortex distribution. In real wakes this distribution may be generally assumed a stochastic parameter, as for the time being, there is no evidence which would link the timing of the generation of stator wake vortices with the relative stator-rotor location. That means that consecutive segments of the same wake shed from a stator blade may behave in a different way in corresponding rotor passages. Or, in other words, consecutive segments of identical wakes shed from consecutive identical stator blades may behave in a different way in one and the same rotor passage.

\section{NUMERICAL AND EXPERIMENTAL VALIDATION}

The best way to validate the correctness of the above presented VDT results would be to compare them with the experiment, preferably performed in the same geometrical and flow conditions. Unfortunately, this way of validation is not possible in the present case, as the examined stage was
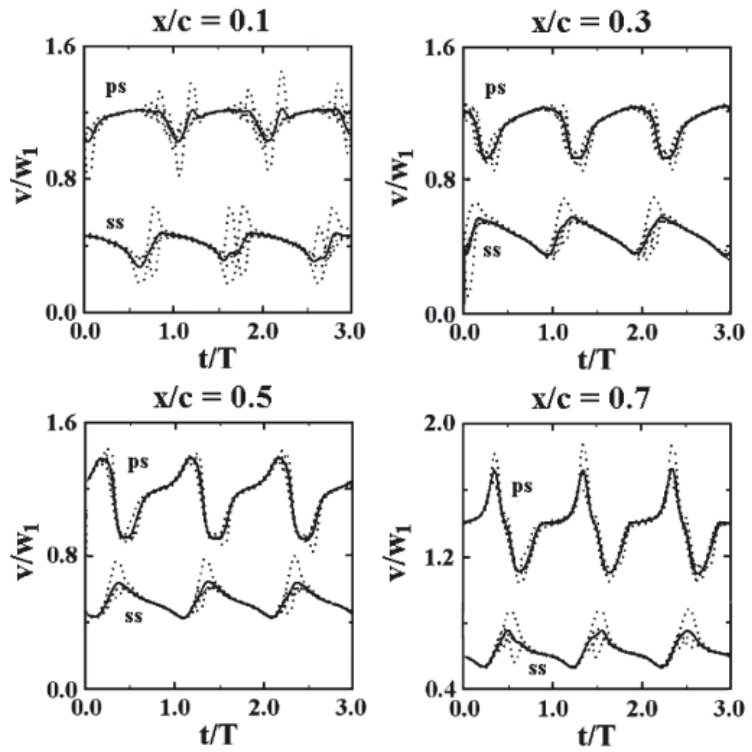

Fig. 11. Velocity fluctuations calculated for the passing vortex wake at selected points inside the rotor passage: dashed lines - individual realisations, continuous line - averaged realisation; $\mathbf{p s ,}$ ss - rotor blade pressure and suction side, respectively 
part of a real turbine in operation in a Polish power plant. Instead, an indirect method was applied which made use of the data recorded in other, but qualitatively similar conditions. It is noteworthy that despite a relatively large number of both numerical and experimental studies on $\mathrm{W} / \mathrm{R}$ interaction which were recently published, finding appropriate data in which the wake development analysis goes as deep as to the vortex structure of the wake was extremely difficult. The most comprehensive and most valuable analysis from the point of view of the present validation was published by Kost et al [33]. Its authors presented the results obtained in a singlestage model turbine in the form of time-histories of velocity fluctuations recorded at points located inside the rotor passage
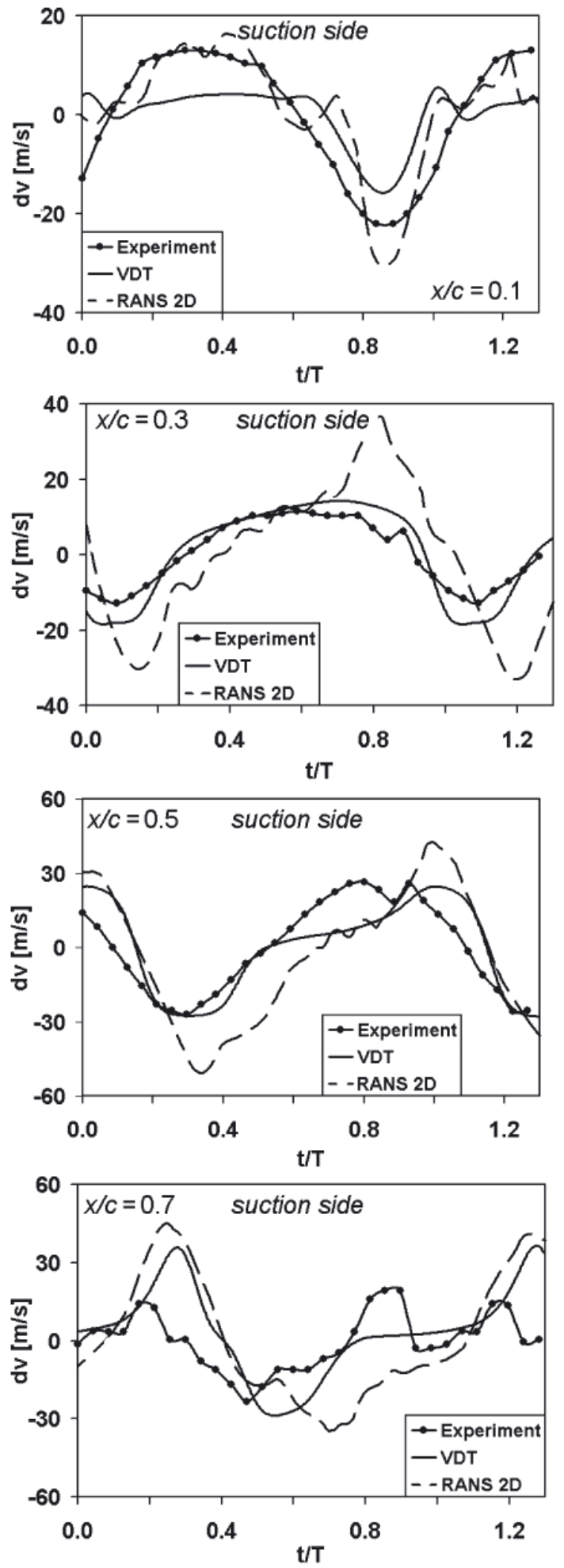

at selected dimensionless distances $\mathrm{x} / \mathrm{c}$ from the rotor blade leading edge and at a distance equal to $1 / 16$ of the rotor pitch from the blade surface, slightly off the rotor blade boundary layer. The experimental time-histories of velocity fluctuations presented by Koch et al. were averaged over 20 realisations. To provide better conditions for comparison, the VDT curves were also averaged, this time over 4 available realisations of the phase shift, $\varphi=0.00,0.25,0.50$ and 0.75 , as shown in Fig. 11 in which the dashed lines represent individual realisations while the continuous lines show the result of averaging.

Figure 12 compares the averaged VDT curves from Fig. 11 with the time-histories presented by Kost et al. Due to different steady-flow velocity levels, the curves shown in the diagrams
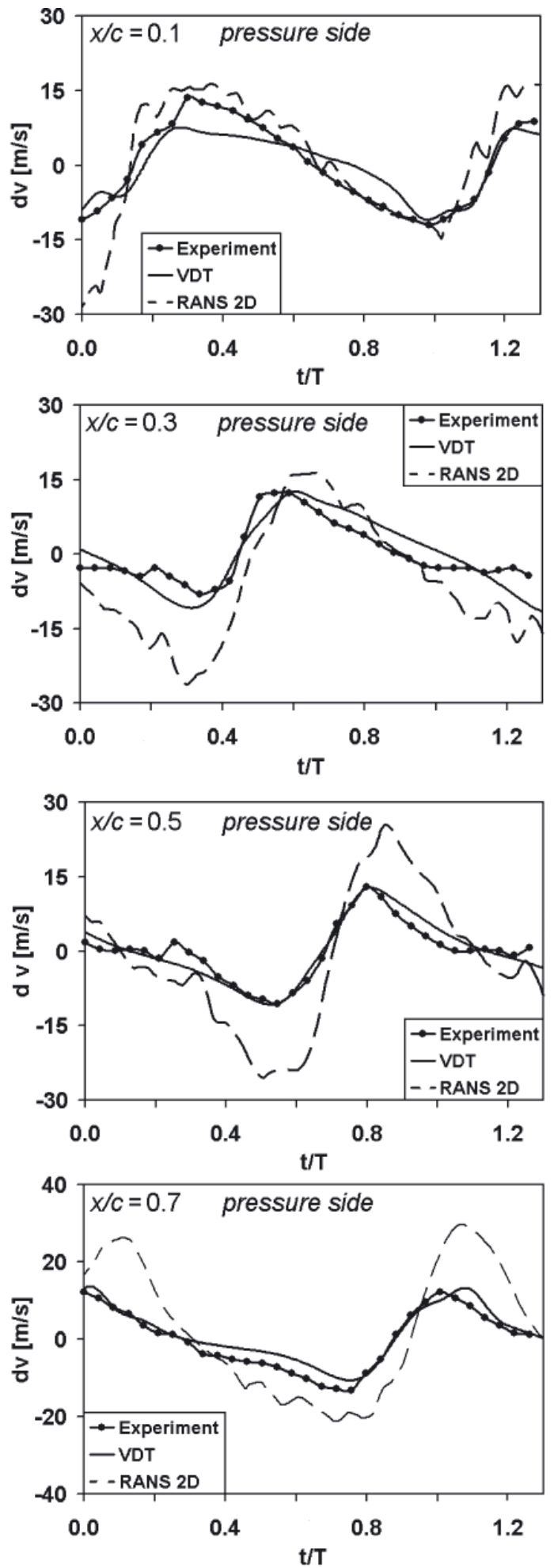

Fig. 12. Velocity fluctuations inside the rotor passage: numerical results vs. experiment 
represent velocity increments, $\mathrm{dv}$, from an average level and not the absolute values. In each diagram the continuous line shows the averaged VDT time-history, while the two remaining curves represent the results presented by Kost et. al. The curve with thickened dots shows the experimental result and the dashed line - RANS calculations performed on 2D grid (only one realisation, without averaging).

When comparing the experimental and VDT curves in successive diagrams we can notice high similarity of their patterns, especially at points located close to the pressure side of the rotor blade. Larger differences are observed at points situated close to the suction side, especially in diagrams for $\mathrm{x} / \mathrm{c}=0.1$ and 0.7 . Relatively high differences in the intensity of velocity fluctuations observed at point $\mathrm{x} / \mathrm{c}=0.1$ may result from insufficient accuracy with which the vorticity distributed in the linear wake is modelled by a sequence of point vortices. When the wake deforms, these differences decrease significantly and only at point $\mathrm{x} / \mathrm{c}=0.7$ remarkable differences can be observed again. This time they are of both quantitative scale referring to the amplitude of fluctuations, and qualitative nature observed in different shapes of the curves being the object of comparison. It is difficult to indicate clearly possible reasons of those differences, which would originate, for instance, from different exit flow conditions, which especially concerns flow angles calculated and recorded in the experiment. This suggestion is made more reliable by the fact that the results of the 2D RANS calculations reveal similar differences to those observed in the VDT time-histories.

\section{EFFECTS OF WAKE DEFORMATION IN VDT AND RANS FORMULATION}

The analysis described in the previous sections delivers a relatively clear and consistent pattern of the $\mathrm{W} / \mathrm{R}$ interaction as an unsteady process controlled by the initial distribution of stator wake vortices at the time when the wake is cut apart by the rotor blades. Different distributions of these vortices provoke differences in their further behaviour and different effects of their interaction with each other and rotor blades.

When confronting this interaction pattern with the RANS procedure of calculations, a basic question can be formulated whether and to which extent the RANS calculations, generally having the form of a single realisation, can be considered as representative for the general $W / R$ interaction process. This question becomes even more important when we keep in mind that the RANS calculations, even performed in unsteady mode, provide rather limited opportunities for re-constructing the vortex structure of the stator wake inside the rotor cascade. Except special cases of integer stator/rotor blade number ratios, this reconstruction would require using the calculation region which covers all passages in stator and rotor rows. When we take into account the grid resolution required for proper capturing of the vortex interaction phenomena, meeting this conditions goes well beyond the calculating potential of even biggest computers in the world. In unsteady RANS calculations the above problem is usually solved using a special periodicity condition, bearing the name of phase lag, which allows the entire calculation region to be reduced to one stator and one rotor passage. Between the stator and rotor cascade a sliding plane is placed, on which the phase lag condition is met. When the wake is treated in a classical way, as a uniform structure shown in Fig. 4, the phase lag condition allows instantaneous wake parameters to be effectively passed between the stator and rotor calculation domains. Unfortunately, the vortex structure of the wake is lost in this process due to a conflict, in general, between the phase shift of the wake vortices and the phase lag defining the instantaneous position of the rotor with respect to the stator blades. As a consequence, the wake approaching the rotor calculation area is an averaged effect of various realisations representing different vortex phase shifts. In this situation the above formulated question can be rephrased to whether the wake "numerically averaged" at the inlet to the rotor calculation domain will behave in the same way, i.e. generate the same time-averaged effects as the real wake in multiple realisations.

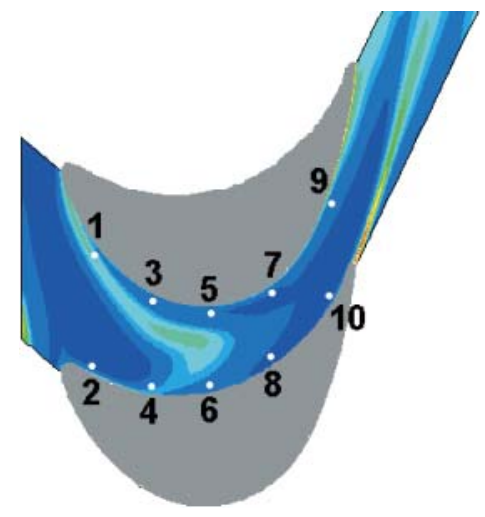

Fig. 13. Locations of recording points inside the rotor passage

In order to find an answer to this question, comparison was made between velocity fluctuations generated by RANS and VDT codes at ten points situated close to the rotor blade trailing edge, Fig. 13. The dimensionless distances $\mathrm{x} / \mathrm{c}$ of these points from the rotor blade leading edge were equal to 0.1 ; $0.3 ; 0.5 ; 0.7$ and 0.9 , while the distance from the blade surface was equal to $1 / 16$ of the rotor pitch. The RANS calculations were performed in two variants differing by grid resolution. The first variant used the grid with 337792 nodes $(6032$ nodes in the circumferential plane $\mathrm{y} 0 \mathrm{z}$ ). This grid resolution can be considered similar to those used in turbine stage flow calculations performed for design purposes. The parameters of the second grid equalled to 698880 nodes in the rotor passage (13 440 in the circumferential plane y0z) and were selected according to recommendation given by Arnone et al [18].

The results of the comparison are shown in Fig. 14. Dashed curves represent coarse-grid RANS calculations (variant 1) while those with large dots were obtained from the fine-grid calculations (variant 2). The third curve represents the VDT calculations used as the reference.

As was shown in Fig. 9, the stator wake inside the rotor passage tends to take a trajectory close to the suction side of the rotor blade. That is why the velocity fluctuations recorded at the pressure side, points $2,4,6,8$ and 10, i.e. at a distance from the wake trajectories, are more regular and very similar to each other. What is also noticeable, the RANS results obtained for the fine grid are closer to the VDT curves than those obtained for the coarse grid. The observed differences between RANS and VDT curves seem to be accidental and do not reveal any regular tendency. Average velocities at the same points are slightly higher in RANS than in VDT calculations.

In the vicinity of the suction side of the rotor blade, points 1, 3, 5, 7 and 9, the differences in the shapes of the RANS and VDT curves are more pronounced. Paradoxically, the largest differences can be observed in the leading section located close to the rotor passage inlet. Downstream, the shapes of the corresponding curves become closer to each other. Like for the pressure side, the RANS results obtained on the fine grid are closer to the VDT results than those obtained on the coarse grid.

Special attention should be paid to the results recorded at point 9. This point is located near the rotor passage exit, close to the 

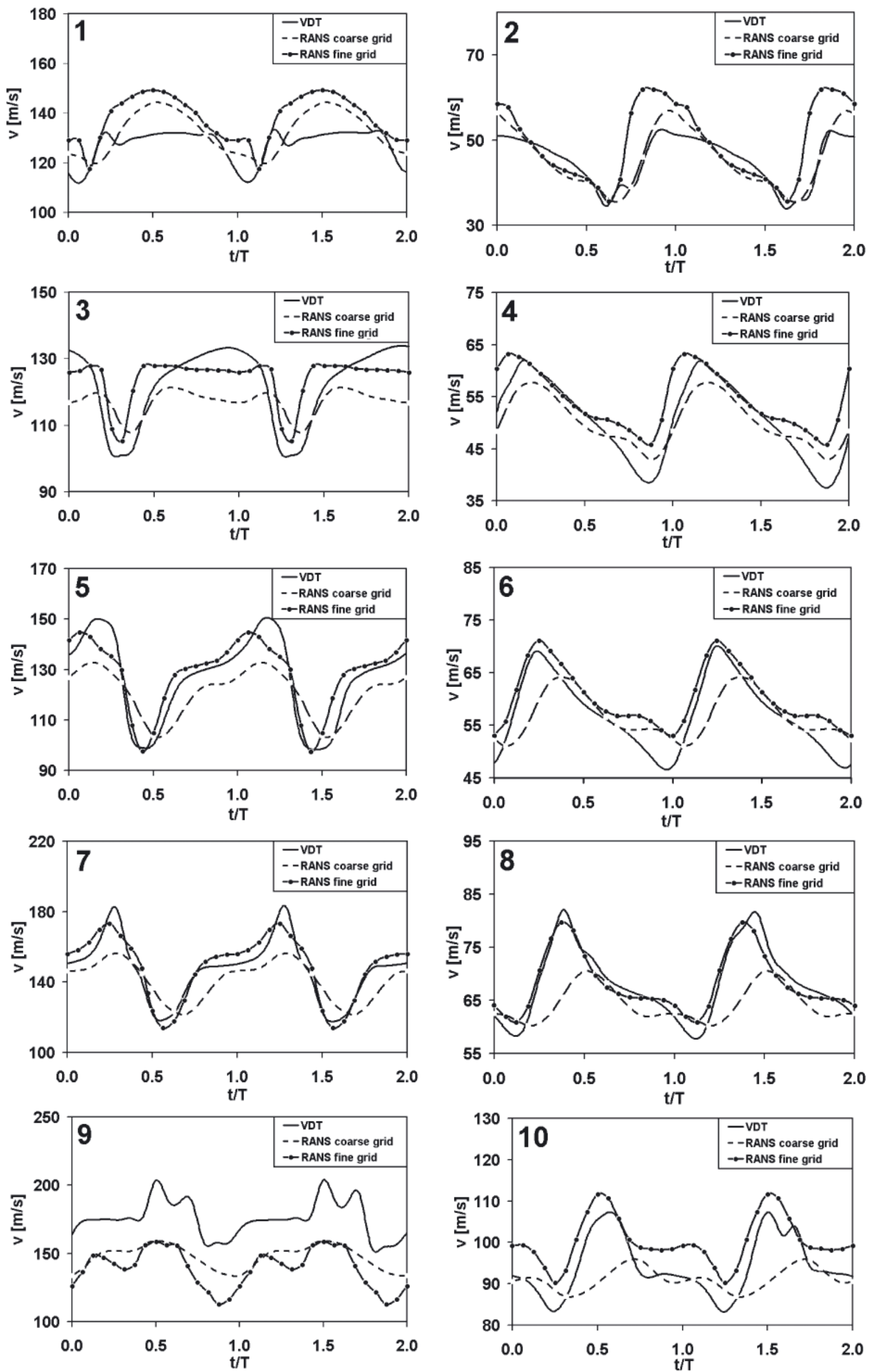

Fig. 14. Velocity fluctuations inside the rotor passage: the effect of grid resolution 
rotor blade suction side, and close to the hypothetical trajectories of vortices composing the stator wake. In these circumstances, all physical differences between the RANS and VDT approaches to modelling the $\mathrm{W} / \mathrm{R}$ interaction, and all numerical errors that cumulate during the step-by-step calculations are expected to be the most remarkable at this point. However, the RANS curve obtained on the fine grid reveals a visibly similar pattern to that shown in the VDT calculations. Moreover, comparable locations and intensities of local extremes are also observed.

The most remarkable difference between RANS and VDT results at point 9 is the average velocity level, visibly lower in the RANS calculations. The reason for this difference results from the fact that the boundary layer is thicker in this passage area and the recording point, unlike all other points, is situated inside it.

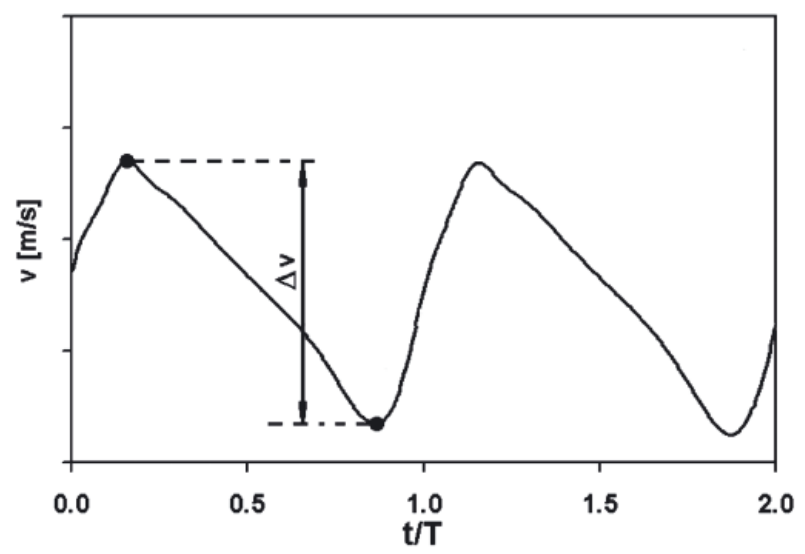

Fig. 15. Definition of velocity fluctuation amplitude

The velocity fluctuation curves observed for coarse-grid RANS calculations suggest that in this case any quantitative assessment of the global effects of the W/R interaction on stage performance (forces, losses, efficiency) can be burdened with rather substantial error.
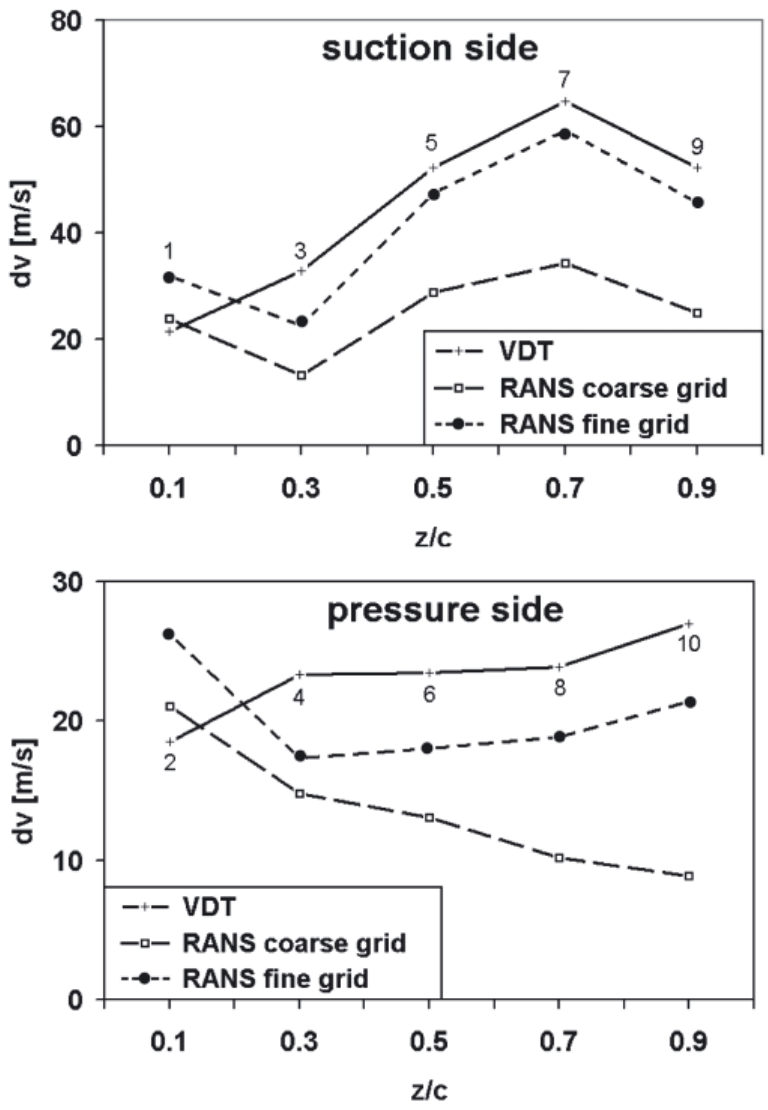

Fig. 16. Velocity fluctuation amplitudes recorded along the rotor passage
To assess the correctness of modelling the rate of stator wake dissipation in the 3D RANS model, velocity fluctuation amplitudes were calculated at the rotor passage points from Fig. 13 in the way illustrated in Fig. 15. The results of these calculations, shown in Fig. 16, reveal that increasing the grid resolution in RANS calculations leads to considerable reduction of wake dissipation. This tendency is illustrated by higher consistency of the amplitude change pattern obtained from RANS calculations with that representing the VDT solution, in which the wake dissipation is not directly taken into account.

Good agreement between the VDT and RANS (fine grid) results shown in Figs. 14 and 16 is highly valued as a definitely positive answer to the question asked at the beginning of this chapter. The RANS calculations, performed on a sufficiently fine grid and making use of a "numerically averaged" stator wake, correctly predict time averaged effects of the real course of W/R interaction.

\section{CONCLUSIONS}

The article discusses the interaction between the stator wakes and the rotor cascade (the $\mathrm{W} / \mathrm{R}$ interaction) in a turbine stage. Taking into account most recent result of both experimental and numerical studies of the inner structure of the wake, the stator wakes were modelled as segments of von Karman vortex streets. To achieve sufficiently high accuracy of unsteady calculations presenting consecutive stages of the W/R interaction, the rotor cascade was represented by an infinite row of blade profiles, the flow through which was calculated using the potential flow with vortex singularities and the conformal mapping method. Geometrical parameters of the stator wake and inlet conditions for the rotor flow were reconstructed from the preliminary 3D RANS calculations done for a stage, the geometry and flow conditions of which were taken from a real high pressure turbine in operation in a Polish power plant. The study has demonstrated that the deformation of the stator wake on its way through the rotor cascade leads to the segregation of two groups of one-sign vortices which then move down the passage along trajectories situated, as a rule, close to the rotor blade suction side. Different phase shifts in the initial distribution of vortices in the stator wake result in noticeable differences in the wake deformation course.

The course of deformation predicted by the VDT analysis was compared to that observed experimentally and analysed numerically by other researchers in a turbine stage. The comparison revealed good qualitative agreement both with respect to particular stages of the wake deformation and its effects, having the form of velocity fluctuations recorded at selected points near the pressure and suction sides of the rotor blade.

The last section of the article analyses the potential of RANS codes for determining quantitative effects of real $\mathrm{W} / \mathrm{R}$ interaction in a turbine stage. The assumed criterion of applicability was the level of agreement of the RANS results with the time-averaged VDT data. It was stated that despite certain differences in the wake structure model used in each of these two codes and the resultant different nature of wake behaviour in the rotor passage: deterministic in RANS calculations and stochastic in VDT calculations, the RANS results obtained on a grid with a sufficiently fine resolution correctly represent the time-averaged effects of the real W/R interaction. The process of averaging of the vortex structure of the wake on the sliding plane between the stator and rotor calculation areas can be considered sort of "numerical averaging" of different realisations of the real vortex W/T interaction. 


\section{REFERENCES}

1. Goldstein R.J., Spores R.A: Turbulent transport on the endwall in the region between adjacent turbine blades, ASME J. Heat Transfer, vol. 110, pp. 862-869, 1988.

2. Doerffer P., Rachwalski J., Magagnato F.: Numerical Investigation of the Secondary Flow Development in Turbine Cascade, TASK Quarterly, vol. 5, pp. 165-178, 2001.

3. Dawes, W. N.: Current \& Future Developments in Turbomachinery CFD, Proceedings, $2^{\text {nd }}$ European Conference on Turbomachinery - Fluid Dynamics and Thermodynamics, Antwerpen, March 5-3, 1997.

4. Meyer, R. X.: The Effect of Wakes on the Transient Pressure and Velocity Distributions in Turbomachines, ASME Journal of Basic Engineering, vol. 80, pp. 1544-1552, 1958.

5. Smith, L. H.: Wake Dispersion in Turbomachines, ASME Journal of Basic Engineering, vol. 88, September, pp. 688-690, 1966.

6. Prandtl L.: Über die Entstehung von Wirbeln in der idealen Flussigkeit, mit Anwendung auf die Tragflugelntheorie und andere Aufgaben, In Gesammt. Abhandl. T. II, Springer-Verlag Berlin Gottingen Heidelberg, 1961, S. 696-513, 1924.

7. Lienhart, W.: Berechnung der instationären Strömung durch gegeneinander bewegte Schaufelgitter und der Schaufelkraf tschwankungen, VDI-Forschungsheft, vol. 562, VDI Verlag, Düsseldorf, 1974.

8. Krammer, P.: Computation of Unsteady Blade Forces in Turbomachines by Means of Potential Flow Theory and by Simulating Viscous Wakes, ASME Paper 82-GT-198, 1982.

9. Korakianitis, T.: On the Propagation of Viscous Wakes and Potential Flow in Axial-Turbine Cascades, ASME Journal of Turbomachinery, vol. 115, pp.118-127, 1993.

10.Hodson, H. P., and Dawes, W. N.: On the Interpretation of Measured Profile Losses in Unsteady Wake-Turbine Blade Interaction Studies, ASME Journal of Turbomachinery, vol. 120, pp. 276-284, 1998.

11.Michelassi, V., Martelli, F., Dénos, R., Arts, T., and Sieverding, C. H.: Unsteady heat Transfer in Stator-Rotor Interaction by Two-Equation Turbulence Model, ASME Journal of Turbomachinery, vol. 121, pp. 436-447, 1999.

12.Solomon, W. J., Walker, G. J., and Hughes, J. D.: Periodic Transition on Axial Compressor Stator: Incidence and Clocking Effects: Part II - Transition Onset Predictions, ASME Journal of Turbomachinery, vol. 121, pp. 408-415, 1999.

13.Dénos, R., Arts, T., Paniagua, G., Michelassi, V., and Martelli, F.: Investigation of the Unsteady Rotor Aerodynamics in a Transonic Turbine Stage, ASME Journal of Turbomachinery, vol. 123, pp. 81-89, 2001.

14.Sieverding, C. H., and Heinemann, H.: The Influence of Boundary Layer State on Vortex Shedding From Flat Plates and Turbine Cascades, ASME Journal of Turbomachinery, vol. 112, pp. 181-187, 1990.

15.Cicatelli, G., and Sieverding, C. H.: The Effect of Vortex Shedding on the Unsteady Pressure Distribution Around the Trailing Edge of a Turbine Blade, ASME Journal of Turbomachinery, vol. 119, pp. 810-819, 1997.

16.Mensink, C.: Numerical Prediction of Periodic Vortex Shedding in Subsonic and Transonic Turbine Cascade Flows, International Journal for Numerical Methods in Fluids, vol. 22, pp. 881-897, 1996.

17.Currie, T. C., and Carscallen, W. E.: Simulation of Trailing Edge Vortex Shedding in a Transonic Turbine Cascade, ASME Journal of Turbomachinery, vol. 120, pp. 10-19, 1998.
18.Arnone, A., Marconcini, M., and Pacciani, R.: On the Use of Dual Time Stepping in Unsteady Turbomachinery Flow Calculations, ERCOFTAC Bulletin No. 42, September, pp. $37-$ 42, 1999.

19.Magagnato, F.: Unsteady flow past a turbine blade using non-linear two-equation turbulence models, Proceedings III European Conference on Turbomachinery: Fluid Dynamics and Thermodynamics, London, March, pp. 221-230, 1999.

20.Lin, C. C.: On the Motion of Vortices in Two Dimensions, University Toronto Press, Toronto, Canada, 1943.

21.Basu, B.C.; Hancock, G.J.: The unsteady motion of a twodimensional airfoil in incompressible inviscid flow, Journal of Fluid Mechanics, vol. 87, pp. 159-178, 1978.

22.Prosnak, W. J.: Computation of Fluid Motions in Multiply Connected Domains, Wissenschaft + technik series, G. Braun, Karlsruhe, Germany, 1987.

23.Milne-Thomson, L.M.: Theoretical Hydrodynamics, London, III edition, 1968.

24. Swirydczuk, J.: A numerical analysis of changes in the conditions of the flow over two identical airfoils caused by the passing vortex structure (in Polish), Reports of the PAS Institute of Fluid-Flow Machinery, No. 94, pp. 3-17, 1992.

25.Swirydczuk, J.: Modelling the interaction of an isolated free vortex with a finite cascade of airfoils with the aid of conformal mapping, Reports of the PAS Institute of Fluid-Flow Machinery, No. 94, pp. 95-111, 1992.

26. Swirydczuk, J.: The Flow Through a Blade Cascade With a Local Shape Deformation, ZAMM, 77, vol. 5, pp. 399-402, 1997.

27. Swirydczuk J.: Vortex Dynamics of the Stator Wake - Rotor Cascade Interaction, ASME Journal of Fluids Engineering, vol. 124, pp. 400-412, 2002.

28.Swirydczuk, J.: The interaction of vortex structures with the approached profiles, (in Polish) D.Sc. thesis, Scientific Reports of the Institute of Fluid-Flow Machinery, No. 533/1492, 233 p, 2004.

29.Swirydczuk, J.: CFD modelling of turbine stage stator/rotor interaction, TASK Quarterly, vol. 10, No. 2, pp. 113-124, 2006.

30.Swirydczuk J.: Analysing Stator/Rotor Interactions in Rotating Machines, Awiacijonno-Kosmiczeskaja Technika i Technołogija, vol. 8/44, pp. 116-120, 2007.

31.Sarpkaya, T.: Computational Methods With Vortices - The 1988 Freeman Scholar Lecture, ASME Journal of Fluids Engineering, vol. 111, pp. 5-52, 1989.

32.Karman, T. von, and Rubach, H.: Über den Mechanismus des Flüssigkeits- und Luftwiderstandes, Physikalische Zeitschrift, vol. 13, pp. 49-59, 1912.

33.Kost, F., Hummel, F., and Tiedemann, M.: Investigation of the Unsteady Rotor Flow Field in a Single HP Turbine Stage, Proceedings, ASME TURBO EXPO 2000, May 8-11, Munich, Germany, 2000.

\section{CONTACT WITH THE AUTHOR}

Jerzy Świrydczuk, Prof. Institute of Fluid-Flow Machinery Polish Academy of Sciences Fiszera 14 80-952 Gdansk, POLAND e-mail: jsk@imp.gda.pl 